\title{
Charge ratchet from spin flip: Space-time symmetry paradox
}

\author{
Sergey Smirnov, ${ }^{1}$ Dario Bercioux, ${ }^{2}$ Milena Grifoni, ${ }^{1}$ and Klaus Richter ${ }^{1}$ \\ ${ }^{1}$ Institut für Theoretische Physik, Universität Regensburg, D-93040 Regensburg, Germany \\ ${ }^{2}$ Freiburg Institute for Advanced Studies (FRIAS) and Physikalisches Institut, Universität Freiburg, D-79104 Freiburg, Germany
}

(Received 10 September 2009; revised manuscript received 9 October 2009; published 16 November 2009)

\begin{abstract}
Traditionally the charge ratchet effect is considered as a consequence of either the spatial symmetry breaking engineered by asymmetric periodic potentials, or time asymmetry of the driving fields. Here we demonstrate that electrically and magnetically driven quantum dissipative systems with spin-orbit interactions represent an exception from this standard idea. In contrast to the so far well established belief, a charge ratchet effect appears when both the periodic potential and driving are symmetric. We show that the source of this paradoxical charge ratchet mechanism is the coexistence of quantum dissipation with the spin-flip processes induced by spin-orbit interactions.
\end{abstract}

DOI: 10.1103/PhysRevB.80.201310

\section{INTRODUCTION}

A system of particles in a periodic potential and driven by a time-dependent external force may exhibit a net current even if the force has zero time average. This so-called particle ratchet effect ${ }^{1-7}$ is used e.g., in nanogenerators of direct charge currents. ${ }^{4,7}$ To excite the particle ratchet current it is traditionally believed that the asymmetry of either the periodic potential or driving force is a must. In the quantum regime a more stringent conclusion has been obtained: in quantum systems in which charged particles populate only one Bloch band the charge ratchet effect does not exist, even if the periodic potential is asymmetric, unless time asymmetry is provided by the driving field. ${ }^{89}$ Indeed, the ratchet effect exists in a single-band system which is driven by a field with harmonic mixing. ${ }^{9,10}$

The concepts and conclusions mentioned above are based on considering particles as spinless, that is, without taking into account any possible impact from switching between the spin states of the particles involved in ratchet transport. In various physical systems there is a plenty of ways to change the spin states of a particle. In this Rapid Communication we limit ourselves to semiconductor heterostructures with spinorbit interactions since from the practical point of view these systems are attractive for fabrication of nanodevices.

For semiconductor heterostructures with spin-orbit interactions, described for example by Rashba ${ }^{11}$ or Dresselhaus ${ }^{12}$ spin-orbit Hamiltonians, the spin ratchet effect is rooted in an asymmetric excitation of spin dynamics by the orbital dynamics induced by an electric field. For electrically driven coherent and dissipative systems with Rashba spin-orbit interaction (RSOI) the spin ratchet mechanism has been confirmed. ${ }^{13-15}$ Even for symmetric periodic potentials and symmetric driving the spin ratchet effect exists. ${ }^{13}$ However, the charge ratchet effect is absent in both the coherent and dissipative cases when both the periodic potential and driving force are symmetric. This could deepen the impression that a system with symmetric periodic potentials will never respond to time-symmetric external fields via the charge ratchet mechanism and systems with spin-orbit interactions like all other systems obey this habitual rule. The present work reveals that this is a delusion and in reality systems with spin-orbit interactions provide a unique opportunity to answer the fundamental questions related to the role of sym-
PACS number(s): 72.25.Dc, 03.65.Yz, 73.23.-b, 05.60.Gg

metries in the charge ratchet phenomena in general.

In this Rapid Communication we show that the space asymmetry of the periodic potentials and the time asymmetry of the driving fields, usually required as key properties of charge ratchets, are not necessary as the Rashba spin-flip processes alone are sufficient even if a dissipative system is time-symmetrically driven. Specifically, it is found that the charge ratchet effect in this case exists for space-symmetric periodic potentials and time-symmetric driving by electric and magnetic fields. It stems just from the simultaneous presence of quantum dissipation and the spin-flip processes of Rashba electrons. The ratchet charge current in the system is unusual. Its queerness consists in the fact that this current, in contrast to early predictions for systems without spin-orbit interactions, ${ }^{8,9}$ appears even when only one energy band provides electrons for transport and no harmonic mixing is present in the driving fields. This charge current is of pure spin-orbit nature and, as a result, it disappears when the spinorbit coupling strength vanishes. Therefore such spin-orbit charge currents can be controlled by the same gate voltage which controls the strength of the spin-orbit coupling in the system. It is evident that this peculiarity of the charge ratchet current is very attractive from the experimental point of view.

The Rapid Communication is organized as follows. Section II presents the model which is solved in Sec. III and numerically analyzed in Sec. IV. Section V concludes the Rapid Communication.

\section{MODEL}

An archetype of the device under investigation is shown in Fig. 1. In this system noninteracting electrons are confined in a quasi-one-dimensional (quasi-1D) periodic structure obtained by appropriately placed gates applied to a twodimensional electron gas (2DEG) with RSOI. The system interacts with an external environment (or bath): the longitudinal orbital degree of freedom of each electron is coupled to orbital degrees of freedom of the external environment. This coupling is the source of dissipation in the system. The electrons are driven by longitudinal electric and transverse inplane magnetic homogeneous fields which are timesymmetric and time-periodic functions with zero mean value. 


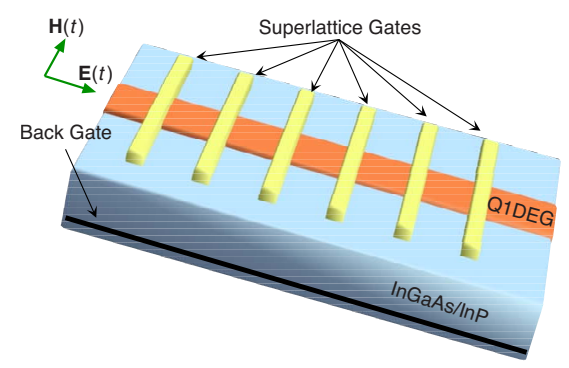

FIG. 1. (Color online) A 2DEG with RSOI of strength $\alpha=9.94 \times 10^{-12} \mathrm{eV} \cdot \mathrm{m}$ is obtained by a gate voltage applied to an InGaAs/InP heterostructure using the "back gate." The electron effective mass is $m=0.037 m_{0}$ with $m_{0}$ being the free electron mass and the effective gyroscopic factor is $g^{*}=-15$. A parabolic confinement of strength $\hbar \omega_{0}=0.225 \mathrm{meV}$ forms in the 2DEG a quasi-onedimensional electron gas. The superlattice with period $L=0.25 \mu \mathrm{m}$ is shaped by the "superlattice gates" which create a symmetric periodic potential. The system is driven by a longitudinal electric field $\mathbf{E}(t)$ and by a transverse magnetic field $\mathbf{H}(t)$ which are time symmetric.

To perform a quantitative analysis of the charge ratchet effect we model the system by the Hamiltonian $\hat{H}(t)=\hat{H}_{0}+\hat{H}_{\mathrm{D}}(t)+\hat{H}_{\mathrm{B}}$, where $\hat{H}_{\mathrm{D}}(t) \equiv-e E(t) \hat{x}-g \mu_{\mathrm{B}} H(t) \hat{\sigma}_{z}$ is the driving term, $\hat{H}_{\mathrm{B}}$ is the bath term of the Caldeira-Leggett model ${ }^{16,17}$ taking into account the orbital coupling between the electron longitudinal degree of freedom, $\hat{x}$, and orbital degrees of freedom of the bath. All properties of the bath are encapsulated in its spectral density $J(\omega)$. Finally, $\hat{H}_{0}$ is the Hamiltonian of the isolated system,

$$
\hat{H}_{0} \equiv \frac{\hbar^{2} \hat{\mathbf{k}}^{2}}{2 m}-\frac{\hbar^{2} k_{\mathrm{sO}}}{m}\left(\hat{\sigma}_{x} \hat{k}_{z}-\hat{\sigma}_{z} \hat{k}_{x}\right)+V(\hat{x}, \hat{z}),
$$

where $V(x, z) \equiv m \omega_{0}^{2} z^{2} / 2+U(x)$ and $U(x)=U(-x)$. In this model it is assumed that the 2DEG is in the $x-z$ plane and the quasi-1D structure is formed along the $x$-axis using a harmonic confinement of strength $\omega_{0}$ along the $z$ axis. The electron-spin $g$-factor is denoted as $g$ and $\mu_{\mathrm{B}}$ is the Bohr magneton. The superlattice period is $L, U(x+L)=U(x)$. The parameter $k_{\mathrm{so}} \equiv \alpha m / \hbar^{2}$ characterizes the strength of the spinorbit coupling.

The electric driving is given by the vector $\mathbf{E}(t)=[E(t), 0,0]$ while the magnetic driving is $\mathbf{H}(t)=[0,0, H(t)]$. We consider the symmetric time dependence: $e E(t) \equiv F \cos [\Omega t], H(t) \equiv H \cos [\Omega t]$. The vector potential is chosen using the Landau gauge $\mathbf{A}(t)=[-H(t) y, 0,0]$. Since $y=0$ in the $2 \mathrm{DEG}$, the vector potential is not explicitly present in the model.

We would like to mention that the in-plane electric fields corresponding to $U(x), m \omega_{0}^{2} z^{2} / 2$ and the driving electric field $E(t)$ are assumed to be much weaker than the out-of-plane electric field forming the 2DEG with RSOI. Thus they produce very weak, in comparison with RSOI, spin-orbit interactions which, therefore, may be neglected.

\section{SOLUTION}

Before starting a rigorous exploration one can already anticipate that the magnetic field driving brings a whiff of fresh physics because the spin dynamics can be controlled directly and not only through the spin-orbit interaction mediating between the electric field and electron spins.

To study the charge ratchet effect at low temperatures, when only the lowest Bloch band of the superlattice is populated with electrons, we calculate the charge current averaged over one driving period. This current in the long time limit provides the stationary charge ratchet response of the system. The common eigenstates of $\hat{x}$ and $\hat{\sigma}_{z}$ represent a convenient basis to obtain this response. Because of the discrete eigenvalue structure of $\hat{x}$ (see below) the basis is called the $\sigma$-discrete variable representation $(\sigma$-DVR) basis. The eigenstates are denoted as $|m, j, \sigma\rangle$, where $m=0, \pm 1, \pm 2, \ldots$, and $j$ and $\sigma$ are the transverse mode and spin quantum numbers, respectively. ${ }^{14,15}$ Below, in parallel with our main goal for this Rapid Communication, that is the charge ratchet current, we also provide the results for the spin ratchet current to show that, as in the coherent case, ${ }^{13}$ it also exists in a dissipative system with symmetric periodic potentials and symmetric driving. In the $\sigma$-DVR basis the averaged charge and spin currents have a simple form, ${ }^{14,15}$

$$
\begin{gathered}
J_{\mathrm{C}}=-e \lim _{t \rightarrow \infty} \sum_{m, j, \sigma} x_{m, j} \frac{d}{d t} P_{j, \sigma}^{m}(t), \\
J_{\mathrm{S}}=\lim _{t \rightarrow \infty} \sum_{m, j, \sigma} \sigma x_{m, j} \frac{d}{d t} P_{j, \sigma}^{m}(t) .
\end{gathered}
$$

In Eq. (2) $P_{j, \sigma}^{m}(t)$ is the averaged population at time $t$ of the $\sigma$-DVR state $|m, j, \sigma\rangle$, the quantities $x_{m, j}=m L+d_{j}$ $\left(-L / 2<d_{j} \leq L / 2\right)$ and $\sigma$ are eigenvalues of $\hat{x}$ and $\hat{\sigma}_{z}$ corresponding to their common eigenstate $|m, j, \sigma\rangle$. Note that in Eq. (2) one has to first calculate the sum and only afterwards to take the limit because the operations of taking limit and infinite summation do not commute as it was proven in Refs. 14 and 15. Additionally, the $\sigma$-DVR basis allows the pathintegral formalism to handle the magnetic driving on an equal footing with the standard electric driving since in this basis the whole driving Hamiltonian, $\hat{H}_{\mathrm{D}}(t)$, is diagonal.

In the long time limit the populations $P_{j, \sigma}^{m}(t)$ come from a master equation ${ }^{14,17}$ which is in this case Markovian. An analytical treatment of this rather complicated problem is possible when the dynamics of $P_{j, \sigma}^{m}(t)$ is treated within the first two transverse modes, i.e., $j=0,1$.

For a detailed study we derive the charge and spin currents assuming that the hopping matrix elements between neighboring $\sigma$-DVR states are small. Following the steps thoroughly described in Ref. 15 we obtain

$$
\begin{gathered}
J_{\mathrm{C}}=\frac{2 e L}{I}\left|\Delta_{\uparrow \downarrow}^{01}\right|^{2}\left|\Delta_{\downarrow \uparrow}^{10}\right|^{2}\left(I_{\uparrow \downarrow}^{01, \mathrm{~b}} I_{\downarrow \uparrow}^{10, \mathrm{~b}}-I_{\uparrow \downarrow}^{01, \mathrm{f}} I_{\downarrow \uparrow}^{10, \mathrm{f}}\right), \\
J_{\mathrm{S}}=\frac{2 L}{I}\left(\left|\Delta_{\uparrow \downarrow}^{01}\right|^{4} I_{\uparrow \downarrow}^{01, \mathrm{f}} I_{\downarrow \uparrow}^{10, \mathrm{~b}}-\left|\Delta_{\downarrow \uparrow}^{10}\right|^{4} I_{\uparrow \downarrow}^{01, \mathrm{~b}} I_{\downarrow \uparrow}^{10, \mathrm{f}}\right),
\end{gathered}
$$

where $\Delta_{\sigma^{\prime} \sigma}^{j^{\prime} j} \equiv\left\langle m+1, j^{\prime}, \sigma^{\prime}\left|\hat{H}_{0}\right| m, j, \sigma\right\rangle$ are the hopping matrix elements of the Hamiltonian of the isolated system, Eq. (1), $I \equiv\left|\Delta_{\uparrow \downarrow}^{01}\right|^{2}\left(I_{\uparrow \downarrow}^{01, f}+I_{\downarrow \uparrow}^{10, b}\right)+\left|\Delta_{\downarrow \uparrow}^{10}\right|^{2}\left(I_{\uparrow \downarrow}^{01, b}+I_{\downarrow \uparrow}^{10, f}\right)$, and $\uparrow, \downarrow$ stand for $\sigma=1,-1$, respectively. The effects of both the driving fields 
and quantum dissipation are in the integrals ${ }^{17}$

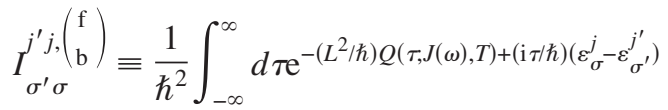

$$
\begin{aligned}
& \times J_{0}\left[\frac{\mp 2 F L+2 g \mu_{\mathrm{B}} H\left(\sigma-\sigma^{\prime}\right)}{\hbar \Omega} \sin \left(\frac{\Omega \tau}{2}\right)\right],
\end{aligned}
$$

where $Q[\tau ; J(\omega), T]$ is the twice integrated bath correlation function,

$$
\begin{aligned}
Q(\tau) \equiv & \frac{1}{\pi} \int_{0}^{\infty} d \omega \frac{J(\omega)}{\omega^{2}}\left[\operatorname{coth}\left(\frac{\hbar \omega}{2 k_{\text {Boltz. }} T}\right)[1-\cos (\omega \tau)]\right. \\
& +\mathrm{i} \sin (\omega \tau)]
\end{aligned}
$$

whose dependence on $\tau$ is fixed by the bath spectral density $J(\omega)$ and temperature $T, \varepsilon_{\sigma}^{j} \equiv\left\langle m, j, \sigma\left|\hat{H}_{0}\right| m, j, \sigma\right\rangle$ are the onsite energies of the isolated system, and $J_{0}(x)$ is the Bessel function of zero order.

Remarkably, Eq. (3) tells us that at low temperatures the ratchet charge and spin transport in the system exists just because of spin-flip processes. Whereas it looks natural for the spin current, it is a quite unexpected and important result for the charge current. This current emerges because the magnetic driving changes the charge dynamics. In this case the spin-orbit interaction plays a role inverse to the one which it plays for the electric driving: the magnetic field exciting spin dynamics induces orbital dynamics through the spin-orbit interaction. The corresponding charge flow, originating just due to the spin-orbit interaction, is finite even when only one Bloch band contributes to transport.

At this point it is important to note that since electrons populate only one Bloch band, the spatial asymmetry is not enough when the driving is time symmetric. This is in complete accordance with the results obtained earlier ${ }^{8,9}$ for spinless particles and is clearly demonstrated in our case by the structure of the Rashba Hamiltonian. Indeed, this Hamiltonian has two terms, $\hat{\sigma}_{z} \hat{k}_{x}$ and $\hat{\sigma}_{x} \hat{k}_{z}$. The first term does not flip the electron spin and does not lead to the charge ratchet effect while the second one flips the electron spin. It is exactly this second term which is responsible for the paradoxical situation: charge ratchet effect for a space-symmetric periodic potential, time-symmetric driving and one Bloch band transport. The charge ratchet effect is exclusively based on the spin-flip processes in the isolated system and thus it is fundamentally different from the charge ratchet mechanisms which have been known so far.

The situation, however, is highly nontrivial and the final conclusions about the existence of the ratchet charge and spin flows cannot be based only on the presence of spin-orbit interactions. There are also external time-dependent fields driving the system and internal quantum dissipative processes. The mutual driving-dissipation effect is incorporated in the integrals [Eq. (4)]. Therefore, a further analysis is required: one should additionally take into consideration the properties of the integrals from Eq. (4) and the properties of the static periodic potential with respect to the spatial inversion symmetry.
This analysis leads to the following results:

$$
\begin{gathered}
F \neq 0, H=0 \quad \Rightarrow \quad J_{\mathrm{C}}=0, J_{\mathrm{S}}=0, \\
F=0, H \neq 0 \quad \Rightarrow \quad J_{\mathrm{C}}=0, J_{\mathrm{S}}=0, \\
F \neq 0, H \neq 0 \quad \Rightarrow \quad J_{\mathrm{C}} \neq 0, J_{\mathrm{S}} \neq 0 .
\end{gathered}
$$

The results presented in Eq. (6) are easily obtained from Eq. (3) if one takes into account that for $U(x)=U(-x)$ the equality $\left|\Delta_{\uparrow \downarrow}^{01}\right|=\left|\Delta_{\downarrow \uparrow}^{10}\right|$ is valid, ${ }^{14,15}$ and for $F=0$ or $H=0$ one makes use of the equality $I_{\sigma^{\prime} \sigma}^{j^{\prime} j, \mathrm{f}}=I_{\sigma^{\prime}, \sigma}^{j^{\prime} j, \mathrm{~b}}$ which follows from Eq. (4).

The principal feature of the physics taking place when $F \neq 0$ and $H \neq 0$ is that the existence of the ratchet effects is not dictated only by properties of the isolated system as in Refs. 14 and 15 . The physical picture is now more intricate. In the charge and spin currents one cannot find clear traces of either driving and dissipation or the isolated system. The two imprints are not separable and the charge and spin ratchet mechanisms are determined by the whole system-plus-bath complex. Note that in comparison with the spin ratchet current in Refs. 14 and 15 the charge ratchet current in Eq. (3) factorizes into two factors in a different way. While in the spin ratchet current in Refs. 14 and 15 there was a factor representing a difference of the hopping matrix elements of the Hamiltonian of the isolated system, now in the charge ratchet current there is a factor representing the difference $I_{\uparrow \downarrow}^{01, \mathrm{~b}} I_{\downarrow \uparrow}^{10, \mathrm{~b}}-I_{\uparrow \downarrow}^{01, \mathrm{f}} I_{\downarrow \uparrow}^{10, \mathrm{f}}$ which is not related only to the isolated system. As one can see from Eq. (4), this difference takes into account the combined effect of dissipation through the twice integrated bath correlation function, driving through the Bessel function and isolated system through the on-site energies storing information about the periodic potential. In the same way as the difference of the hopping matrix elements of the Hamiltonian of the isolated system in Refs. 14 and 15 dictated the existence of the spin ratchet current, now the difference $I_{\uparrow \downarrow}^{01, \mathrm{~b}} I_{\downarrow \uparrow}^{10, \mathrm{~b}}-I_{\uparrow \downarrow}^{01, \mathrm{f}} I_{\downarrow \uparrow}^{10, \mathrm{f}}$ rules the existence of the charge ratchet current in the present Rapid Communication and results in the combined effect of the isolated system, dissipation and driving, as mentioned above. It is important to remember that this combined effect takes place only if the spin-orbit coupling is finite because $\Delta_{\uparrow \downarrow}^{01}=\Delta_{\uparrow \uparrow}^{10}=0$ in the absence of RSOI, as it has been proven in Refs. 14 and 15.

\section{NUMERICAL RESULTS}

Numerical calculations based on Eqs. (3) and (4) have been performed to obtain the dependence of the ratchet currents on $F$ and $H$. Figure 2 shows this dependence. The superlattice is modeled by the symmetric potential $U(x)$ $=2.6 \hbar \omega_{0}[1-\cos (2 \pi x / L)]$. The period is $L=2.5 \sqrt{\hbar / m \omega_{0}}$ which gives $k_{\mathrm{so}} L \approx 0.368 \pi$. The driving frequency of the electric and magnetic fields is $\Omega=\sqrt{3} \omega_{0} / 4$. The bath is Ohmic with the exponential cutoff at $\omega_{c}=10 \omega_{0}: J(\omega)$ $=\eta \omega \exp \left(-\omega / \omega_{c}\right)$. The viscosity coefficient is $\eta=0.1 m \omega_{0}$ and the temperature is $k_{\text {Boltz. }} T=0.5 \hbar \omega_{0}$. As expected from Eq. (6) the ratchet effects exist for the space-symmetric periodic potential and time-symmetric driving. From Fig. 2 one 


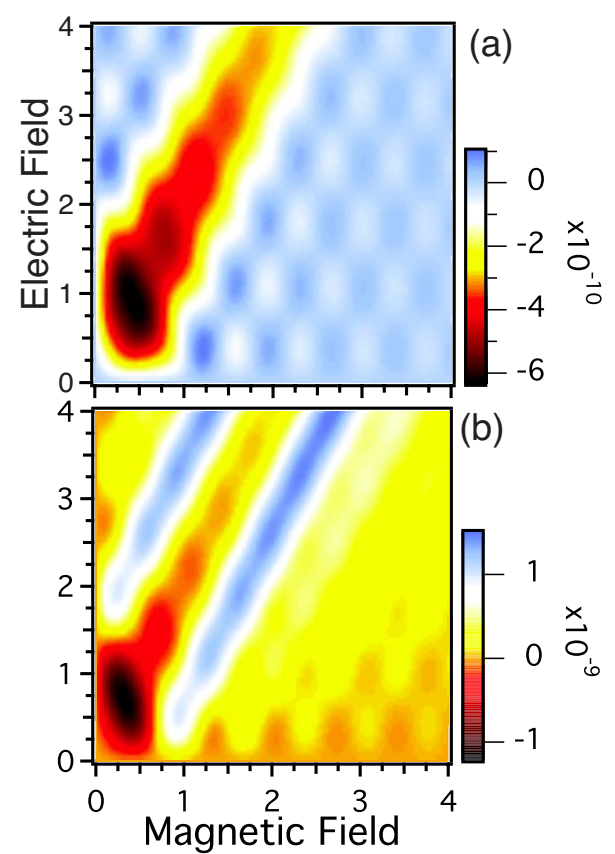

FIG. 2. (Color online) The charge and spin ratchet currents as functions of the amplitudes of the electric and magnetic fields. (a) Charge current. (b) Spin current. The amplitudes of the electric, $F L$, and magnetic, $g \mu_{\mathrm{B}} H$, fields are in units of $\hbar \omega_{0}$. The currents are in units of $L \omega_{0}$. According to Eq. (6) the charge and spin currents are excited when both the electric and magnetic fields simultaneously drive the system.

also observes an oscillatory behavior of the ratchet currents.

These oscillations are detailed in Fig. 3. As one can see the currents can be equal to zero even when both of the driving fields are finite. These zero-current points are not universal: they depend on concrete values of the physical parameters of the isolated system and bath. In contrast, the conditions in Eq. (6) are universal; i.e., they do not depend on concrete values of the physical parameters of the semiconductor heterostructure and environment.

Finally, we would like to note that since our theory is a theory of a strongly dissipative tight-binding system, the charge ratchet current is small but detectable. For example

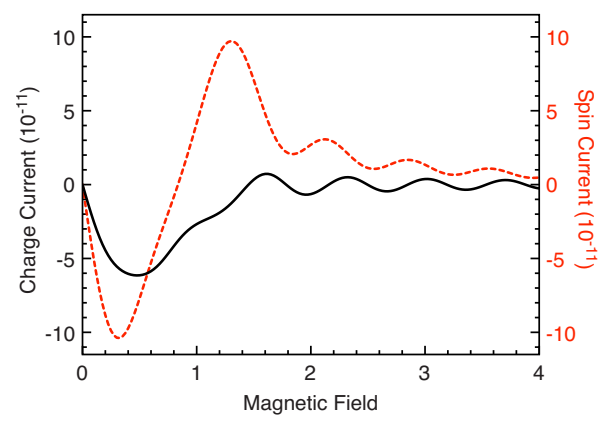

FIG. 3. (Color online) The charge (solid curve) and spin (dashed curve) ratchet currents as functions of the magnetic field amplitude. The magnetic amplitude, $g \mu_{\mathrm{B}} H$, is in units of $\hbar \omega_{0}$. The electric amplitude is fixed, $F L=\hbar \omega_{0}$. The currents oscillate and have nonuniversal zero points which depend on concrete values of the physical parameters of the system-plus-bath complex.

using the parameters of Ref. 15 we get the charge ratchet current $J_{\mathrm{C}} \sim 10 \mathrm{fA}$. We expect that models with weak dissipation or/and weak periodic potentials will give much larger charge ratchet currents in the fully symmetric setup presented in this Rapid Communication.

\section{CONCLUSION}

In summary, in contrast to the common belief, we have shown that the existence of spin-flip processes in a dissipative system is already sufficient to produce the charge ratchet effect even if the periodic potential is space symmetric and the system is driven by time-symmetric fields. To be specific we have considered Rashba spin-orbit interaction as a mechanism for the electron-spin flip. The charge ratchet current has been found to have a purely spin-flip origin. The space asymmetry of the periodic potential and the time asymmetry of the driving fields have not been necessary.

\section{ACKNOWLEDGMENTS}

Support from the DFG under Program No. SFB 689 and Excellence Initiative of the German Federal and State Governments is acknowledged.
${ }^{1}$ R. D. Astumian and P. Hänggi, Phys. Today 55(11), 33 (2002).

${ }^{2}$ P. Reimann, M. Grifoni, and P. Hänggi, Phys. Rev. Lett. 79, 10 (1997).

${ }^{3}$ F. Jülicher, A. Ajdari, and J. Prost, Rev. Mod. Phys. 69, 1269 (1997).

${ }^{4}$ H. Linke, T. E. Humphrey, A. Löfgren, A. O. Sushkov, R. Newbury, R. P. Taylor, and P. Omling, Science 286, 2314 (1999).

${ }^{5}$ J. B. Majer, J. Peguiron, M. Grifoni, M. Tusveld, and J. E. Mooij, Phys. Rev. Lett. 90, 056802 (2003).

${ }^{6}$ P. Hänggi and F. Marchesoni, Rev. Mod. Phys. 81, 387 (2009).

${ }^{7}$ P. Olbrich, E. L. Ivchenko, R. Ravash, T. Feil, S. D. Danilov, J. Allerdings, D. Weiss, D. Schuh, W. Wegscheider, and S. D. Ganichev, Phys. Rev. Lett. 103, 090603 (2009).

${ }^{8}$ M. Grifoni, M. S. Ferreira, J. Peguiron, and J. B. Majer, Phys. Rev. Lett. 89, 146801 (2002).

${ }^{9}$ I. Goychuk and P. Hänggi, Europhys. Lett. 43, 503 (1998).
${ }^{10}$ A. V. Ponomarev, S. Denisov, and P. Hänggi, Phys. Rev. Lett. 102, 230601 (2009).

${ }^{11}$ E. Rashba, Fiz. Tverd. Tela (Leningrad) 2, 1224 (1960) [Sov. Phys. Solid State 2, 1109 (1960)].

${ }^{12}$ G. Dresselhaus, Phys. Rev. 100, 580 (1955).

${ }^{13}$ M. Scheid, A. Pfund, D. Bercioux, and K. Richter, Phys. Rev. B 76, 195303 (2007).

${ }^{14}$ S. Smirnov, D. Bercioux, M. Grifoni, and K. Richter, Phys. Rev. Lett. 100, 230601 (2008).

${ }^{15}$ S. Smirnov, D. Bercioux, M. Grifoni, and K. Richter, Phys. Rev. B 78, 245323 (2008).

${ }^{16}$ A. O. Caldeira and A. J. Leggett, Phys. Rev. Lett. 46, 211 (1981).

${ }^{17}$ U. Weiss, Quantum Dissipative Systems, 3rd ed. (World Scientific, Singapore, 2008). 